\section{Endotyping idiopathic pulmonary fibrosis should improve outcomes for all patients with progressive fibrotic lung disease}

\author{
Gisli Jenkins
}

The first few years of the current millennium has seen a paradigm shift in the way people view idiopathic pulmonary fibrosis (IPF). In 2004, Raghu et al ${ }^{1}$ published the first multicentre, double blind, randomised, placebo-controlled trial in IPF. Although a negative study, it taught the field much about the natural history and conduct of clinical trials in IPF. Ultimately, this study paved the way for the Clinical Studies Assessing Pirfenidone in idiopathic pulmonary fibrosis: Research of Efficacy and Safety Outcomes (CAPACITY) studies ${ }^{2}$ that lead to the approval of Pirfenidone in Europe and eventually the Assessment of Pirfenidone to Confirm Efficacy and Safety in Idiopathic Pulmonary Fibrosis $(\mathrm{ASCEND})^{3}$ and Prospective Observation of Fibrosis in the Lung: Clinical Endpoints (IMPULSIS) $^{4}$ studies, which might lead to approval for the use of two diseasemodifying drugs for the treatment of IPF in both the USA and Europe. Any thought at the turn of the century that progressive fibrotic lung disease, in the absence of inflammation, might be modifiable would have been regarded with a fair degree of scepticism. The field has come far.

However, the journey has only just begun. Extrapolating data from recent epidemiological studies would suggest there has been approximately a doubling of the incidence of IPF and associated healthcare requirements since the turn of the century. ${ }^{5}$ Furthermore, patients enrolled into clinical trials represent a unique subpopulation of patients with IPF, itself a subpopulation of patients with progressive fibrotic lung disease. Clinical trials in IPF have, so far, focused on mild to moderate IPF based on arbitrary lung function definitions of severity and the European Respiratory Society/American Thoracic Society criteria for defining IPF. While this has delivered robust, effective clinical trials, it disenfranchises a large number of patients with

\footnotetext{
Correspondence to Dr Gisli Jenkins, Respiratory Research Unit, Clinical Sciences Building, City Hospital Campus, Nottingham University Hospitals, Hucknall Road, Nottingham NG5 1PB, UK; gisli.jenkins@nottingham.ac.uk
}

progressive fibrosis. Similarly, criteria set by the National Institute of Health and Care Excellence for prescribing pirfenidone in IPF excludes a significant proportion of patients at either end of the disease spectrum. In addition, emerging data from various registries around the world suggest that unclassifiable fibrotic disease is the diagnosis in a significant proportion of patients with interstitial lung disease, even in countries where high rates of biopsies are performed. ${ }^{7}$ Therefore, estimating 25\% of patients in the UK with progressive fibrotic lung disease are unclassifiable and 33\% of the remaining patients with IPF are excluded from current management strategies due to severity criteria, the number of patients for whom there is no available therapy is exactly the same as it was at the start of this millennium.

Strict morphological phenotyping has generated excellent results in interventional clinical trials but this approach reduces clinical generalisability, despite the probability of shared pathogenic mechanisms across a range of progressive fibrotic lung diseases. Furthermore, despite accurate phenotyping there remains considerable heterogeneity in disease progression, survival and response to therapy suggesting a range of endotypes within fibrotic disease phenotypes. Therefore, a new approach needs to be taken. A move towards molecular phenotyping, which has been so effective in oncology, is required and this approach is exemplified by DePianto et $\mathrm{al}^{8}$ in this issue of Thorax. Analysing lung tissue using a combination of genomic and histological techniques the authors identified two patterns of disease in IPF: abnormal bronchiolisation and lymphoid aggregates, which could be targetable by new therapies.

However, the DePianto et $a l^{8}$ recognise the limitations associated with tissue analysis in IPF. Unlike molecular analysis in oncology, tissue accessibility in IPF is problematic because of the high mortality associated with biopsy, ${ }^{9} 10$ the spatial and temporal heterogeneity of the pathological lesions and the excellent diagnostic imaging capabilities of current high-resolution CT.
Therefore, tissue is usually only obtained from patients with a low pretest probability of having IPF or from explanted lung tissue following transplant meaning longitudinal assessment of molecular signals from lung tissue must be obtained by other means. One approach is to use nuclear imaging which can identify molecular signals in lung tissue; ${ }^{11}{ }^{12}$ however, an alternative approach, adopted by DePianto et al, ${ }^{8}$ is to identify soluble mediators that reflect molecular signals identified following immunohistochemical analysis of lung tissue. The strength of such an approach is that these mediators can be easily measured and longitudinally sampled. However, serum sampling is not specific to the lung and could be confounded by comorbid diseases in other organ systems or drugs that alter metabolic functions; similarly, it is not precisely known how changing levels of soluble mediators over time reflects disease activity. Such findings need to be validated in large prospective longitudinal cohorts of patients with IPF such as the PROFILE cohort. $^{13}$

However, despite these limitations this is an exciting approach yielding promising results reflecting established and novel mechanisms of disease. The role of matrix metalloproteinases (MMPs) in IPF has been recognised for some time and MMP7 has been identified as a promising biomarker. ${ }^{14}$ Recently, a role for MMP3 in fibrogenesis, by driving epithelial to mesenchymal transition, has been described. ${ }^{15}$ There is also emerging evidence that the epithelium in fibrotic regions of the lung may not be derived from alveolar type II cells but from the small airways. ${ }^{16}$ This study further supports a concept which should encourage researchers and reviewers to be less rigid about the in vitro approach used to study fibrotic epithelial cells.

CXCL13 is a chemokine involved in B cell trafficking. A recent role for CXCL13 has been described in IPF with high levels in serum associated with IPF compared with healthy controls or patients with COPD. ${ }^{17}$ Furthermore, their data suggested that levels of CXCL13 rose in patients over the course of their disease. ${ }^{17}$ The data in the current study would support those prior observations and together these studies lend weight to further investigating the role of $\mathrm{B}$ cell depletion strategies for the treatment of IPF in appropriately stratified patients.

With the prospect of a number of antifibrotic drugs becoming available in the near future, there is a clear need to stratify patients with progressive fibrotic lung disease. By identifying distinct molecular pathways that may define specific endotypes of disease reflecting rates of disease 
progression, this study raises the possibility that molecular endotyping may be used to stratify patients into those with progressive or stable fibrotic disease or more speculatively those who might respond to B cell depletion therapy. Importantly, these results should promote similar studies in non-IPF fibrotic lung disease to determine whether similar endotypes exist that can predict accelerated fibrogenesis regardless of aetiology. Ultimately, the aim should be to undertake interventional clinical trials on all patients with progressive fibrotic lung disease stratified by the molecular endotype rather than the clinical diagnosis of IPF.

Currently, patients with progressive fibrotic lung disease other than IPF are excluded from the major therapeutic advances that are currently being made. Studies such as those described by DePianto et al offer hope to this large group of patients with lung fibrosis that soon it will be their turn to benefit from advances in the field of fibrosis research.

Competing interests GJ received research grants from GlaxoSmithKline, Novartis and Biogenldec and have provided consultancy for GlaxoSmithKline, Boehringer Ingleheim, Intermune, Medlmmune and Biogenldec.

Provenance and peer review Not commissioned; internally peer reviewed.

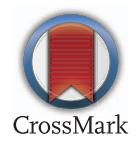

To cite Jenkins G. Thorax 2015;70:9-10.

Published Online First 19 November 2014

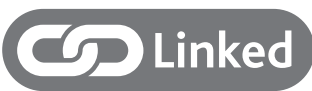

- http://dx.doi.org/10.1136/thoraxjnl-2013-204596

Thorax 2015;70:9-10.

doi:10.1136/thoraxjn-2014-206209

\section{REFERENCES}

1 Raghu G, Brown KK, Bradford WZ, et al. A placebo-controlled trial of interferon gamma- $1 \mathrm{~b}$ in patients with idiopathic pulmonary fibrosis. $N$ Engl J Med 2004;350:125-33.

2 Noble PW, Albera C, Bradford WZ, et al. Pirfenidone in patients with idiopathic pulmonary fibrosis (CAPACITY): two randomised trials. Lancet 2011;377:1760-9.

3 King TE Jr, Bradford WZ, Castro-Bernardini S, et al. A phase 3 trial of pirfenidone in patients with idiopathic pulmonary fibrosis. N Engl I Med 2014;370:2083-92.

4 Richeldi L, du Bois RM, Raghu G, et al. Efficacy and safety of nintedanib in idiopathic pulmonary fibrosis. N Engl J Med 2014;370:2071-82.

5 Navaratnam V, Fleming KM, West J, et al. The rising incidence of idiopathic pulmonary fibrosis in the U.K. Thorax 2011;66:462-7.

6 Navaratnam V, Fogarty AW, Glendening R, et al. The increasing secondary care burden of idiopathic pulmonary fibrosis: hospital admission trends in England from 1998 to 2010. Chest 2013;143:1078-84.

7 Ryerson CJ, Urbania TH, Richeldi L, et al. Prevalence and prognosis of unclassifiable interstitial lung disease. Eur Respir J 2013;42:750-7.
8 DePianto DJ, Chandriani S, Abbas AR, et al. Heterogeneous gene expression signatures correspond to distinct lung pathologies and biomarkers of disease severity in idiopathic pulmonary fibrosis. Thorax 2015;70:48-56.

9 Morris D, Zamvar V. The efficacy of video-assisted thoracoscopic surgery lung biopsies in patients with Interstitial Lung Disease: a retrospective study of 66 patients. J Cardiothorac Surg 2014;9:45.

10 Nguyen W, Meyer KC. Surgical lung biopsy for the diagnosis of interstitial lung disease: a review of the literature and recommendations for optimizing safety and efficacy. Sarcoidosis Vasc Diffuse Lung Dis 2013;30:3-16.

11 John AE, Luckett JC, Tatler AL, et al. Preclinical SPECT/CT imaging of $\alpha \vee \beta 6$ integrins for molecular stratification of idiopathic pulmonary fibrosis. J Nucl Med 2013;54:2146-52.

12 Win T, Thomas BA, Lambrou T, et al. Areas of normal pulmonary parenchyma on HRCT exhibit increased FDG PET signal in IPF patients. Eur I Nucl Med Mol Imaging 2014;41:337-42.

13 Maher TM. PROFILEing idiopathic pulmonary fibrosis: rethinking biomarker discovery. Eur Respir Rev 2013;22:148-52.

14 Rosas IO, Richards TJ, Konishi K, et al. MMP1 and MMP7 as potential peripheral blood biomarkers in idiopathic pulmonary fibrosis. PLoS Med 2008; 5:e93.

15 Yamashita CM, Dolgonos L, Zemans RL, et al. Matrix metalloproteinase 3 is a mediator of pulmonary fibrosis. Am J Pathol 2011;179:1733-45.

16 Seibold MA, Smith RW, Urbanek C, et al. The idiopathic pulmonary fibrosis honeycomb cyst contains a mucocilary pseudostratified epithelium. PLOS ONE 2013;8:e58658.

17 Vuga LJ, Tedrow JR, Pandit KV, et al. C-X-C motif chemokine 13 (CXCL13) is a prognostic biomarker of idiopathic pulmonary fibrosis. Am I Respir Crit Care Med 2014;189:966-74. 